\title{
Discussion of paper of Professor Murnaghan et al.
}

\section{Chairman: DR HACHEN}

Mr GibBon (G.B.). Professor Murnaghan has an ideal opportunity to distinguish between the effect of conus and cauda equina lesions, and whether in fact the changes that we have observed with posture and with thigh-cuffs compression are due to interruption of the sympathetic supply in the conus or whether they are due to withdrawal of the cholinergic function. I hope we will get further information about this at a later stage.

SIR LUDWIG GutTMANN (G.B.). I wonder whether by inserting electrodes you could educate the monkeys to express their bladder like human beings and then find out what happens.

DR BRINDLEY (G.B.). I've done a number of experiments rather like these, using mainly baboons but also a few rhesus monkeys. It is very unfortunate to show pictures of chimpanzees even frivolously when you are talking about rhesus monkeys. Other points of difference are just minor. We find mostly the same things as you but the effects of stimulation of both presacral nerve and pudendal nerve can be very much greater than you have shown. We find that in our few rhesus monkeys as well as in our baboons it is not a species difference, but probably a matter of stimulus parameters. So it is just worth knowing because one can get the urethral pressure profile rising by a factor of 4 or 5 on either presacral or pudendal nerve stimulation.

Chairman. I did not understand how it is possible that both urocholine and atropine can have a lowering effect on urethral pressure. You showed this on your graph. How could both the cholinergic and anticholinergic component bring about the same result?

Professor Murnaghan (G.B.). I'm not sure how this comes about, but you have to take cognisance of the fact that at the end point of whatever you are doing with the central nervous system in the context of the bladder and the urethra, most of the time you have smooth muscle. The most extraordinary thing about smooth muscle is the way in which adrenalin and acetylcholine and atropine and many of these drugs work is to change the distribution of the sodium and potassium across the cellular membrane. I think Dr Edith Bulbring in Oxford showed this a long time ago with isolated strips of muscle that get contrary results by the use of stimulation and relaxing agents on smooth muscle according to the starting tonus. I apologise for the low numbers in these series but we have had less than two animals. The problem with our drug studies is that we cannot go to the pharmacopœia and get the appropriate dosage for these animals so that any drug which had an effect upon the autonomic nervous system would thereby produce reflex changes in the cardiovascular system. We have two special animals with chronic catheters in the vena cava and the aorta. We've used these to obtain our drug dosage responses to carbachol and to atropine. They were repeated but only in two animals. I must apologise for the use of the chimpanzees, Dr Brindley. These were shown frivolously I think to disengage people from the clinical scene because the one thing that ought to be stressed most carefully is that you cannot make a direct observation on an animal and take it directly into your clinical practice, but we hope that one day we will get there.

SIR LUDWIG. It is an interesting point you have mentioned about the various responses of the autonomic system to these kinds of drugs. If you do a cervical sympathectomy the adrenalin will inhibit sweating: however, in a sympathectomised horse it produces sweating. With all these kinds of drugs we have to consider the varying reactions of individuals both in animals and in man.

Chairman. I think it's true that when we extrapolate results in animal experimentation into clinical experience, we require to be extremely careful regarding dosage and interpretation of results.

DR PERKaSh (U.S.A.). One can explain the effect of cholinergics on the bladder because by setting up a micturition reflex a bladder contraction occurs with a drop in urethral pressure. 